\title{
Asociaciones olfativas en torno a la percepción del espacio y el servicio recibido por los asociados a un fondo de empleados en Manizales*
}

\author{
Felipe Antonio Gallego López ${ }^{* *}$ \\ Salomón Mejía Gallo*** \\ Recibido: 2018-09-21. Enviado a pares: 2018-09-28. \\ Aprobado por pares: 2018-11-15. Aceptado: 2018-12-03 \\ https://doi.org/10.22395/angr.v17n34a13
}

\begin{abstract}
Resumen
El presente artículo muestra los resultados obtenidos en la investigación sobre marketing sensorial y su influencia en la experiencia de compra, el cual busca aportar conocimiento al campo del marketing sensorial y su importancia en la construcción de experiencias memorables para los públicos. De esta forma se quiere comprender si un aroma puede relacionarse con la experiencia del servicio recibido en las instalaciones de un fondo de empleados de Manizales por medio de una investigación cuantitativa-descriptiva de alcance correlacional cuasiexperimental.

En la primer semana se indagó en la percepción a 301 personas que ingresaron a las instalaciones del fondo de empleados sin intervención de algún esquema de aroma. Para la segunda semana se hizo una intervención, se recolectaron 301 encuestas a las personas que ingresaron y que calificaron las mismas variables en el espacio ya aromatizado.

Se sistematizaron los cuestionarios en una matriz en Microsoft Excel $2013^{\circledR}$ para, posteriormente, realizar el análisis estadístico con el IBM SPSS v. 21. Se verificó la consistencia interna con alfa de Cronbach de 0,856, se caracterizó la población y se establecieron asociaciones ordinales mediante el coeficiente Gamma de Goodman y Kruskal, adicionalmente se comprobaron con la prueba Chi-cuadrado las posibles asociaciones. Uno de los resultados más importantes del estudio demostró que la estimulación del sentido del olfato puede asociarse de manera positiva en la percepción de las variables de atención en el servicio y limpieza.
\end{abstract}

Palabras clave: marketing; experiencial; sensorial; olfativo; aromas; servicio; persuasión; publicidad; asociaciones.

El presente artículo surgió como producto del proyecto de investigación Marketing sensorial y su influencia en la experiencia de compra, en la línea de sociedad, comunicación publicitaria y desarrollo del Grupo de Investigación Altamira, del programa académico curricular en Publicidad de la Facultad de Ciencias Sociales, Humanidades y Teología de la Universidad Católica de Manizales (UCM).

" Matemático, magíster en Ciencias en Matemática Aplicada, docente asistente de la Universidad Católica de Manizales, docente auxiliar en dedicación de cátedra 0.4 de la Universidad Nacional de Colombia, sede Manizales, asesor estadístico de la Dirección de Investigaciones de la UCM, investigador en las áreas de estadística aplicada a la educación, al marketing, entre otras, miembro del grupo de investigación EFE en la UCM. fagallego@ucm.edu.co. Orcid: http://orcid. org/0000-0002-3653-080X

... Publicista, especialista en Gerencia en Mercadeo y Ventas, docente instructor, investigador en las áreas consumidor, creatividad y conceptualización de campañas publicitarias, miembro del grupo de investigación Altamira, Facultad de Humanidades y Teología de la UCM. smejía@ucm.edu.co. Orcid: https://orcid.org/0000-0001-9451-072X 


\title{
Olfactory associations around space perception and the service received by members of a fund of employees in Manizales
}

\begin{abstract}
This paper shows the results obtained in a research on sensory marketing and its influence on the shopping experience, which seeks to contribute knowledge to the sensory marketing field and its importance in making memorable experiences for the public. In this way it is intended to understand if a particular scent can be related to the experience of the service received in the facilities of fund of employees in Manizales through a quantitative-descriptive research with a quasi-experimental correlational scope.

In the first week, 301 people who entered the facilities of the fund of employees without any scent scheme were interviewed. In the second week, an intervention was made: 301 surveys were collected from people who entered the same facilities, but this time with a scent scheme, and who rated the same variables.

Surveys were systematized in a Microsoft Excel $2013^{\circledR}$ spreadsheet, and the statistical analysis was carried out with the IBM SPSS v. 2.1. software. Internal consistency was verified with Cronbach's alpha of 0.856 . Also, the population was characterized, and ordinal associations were established by the Gamma coefficient of Goodman and Kruskal. In addition, the possible associations were verified with a Chi-square test. One of the most important results of the study showed that smell stimulation can be linked in a positive way in the perception of the attention variables regarding service and cleaning.
\end{abstract}

Keywords: marketing; experiential; sensory; olfactory; scents; service; persuasion; advertising; link.

\section{Associações olfativas em torno à percepção do espaço e do serviço recebido pelos associados a um fundo de apoio aos funcionários em Manizales}

\begin{abstract}
Resumo
O presente artigo mostra os resultados obtidos na pesquisa sobre marketing sensorial e sua influência na experiência de compra, que busca oferecer conhecimento ao campo do marketing sensorial e sua importância na construção de experiências memoráveis para os públicos. Dessa forma, visa-se compreender se um aroma pode estar relacionado com a experiência do serviço recebido nas instalações de um fundo de empregados de Manizales por meio de uma pesquisa quantitativa-descritiva de alcance correlacional quasi-experimental. Na primeira semana, indagou-se a percepção de 301 pessoas que ingressaram nas instalações de um fundo de apoio a funcionários sem intervenção de qualquer esquema de aroma. Na segunda semana, fez-se uma intervenção e entrevistou-se 301 pessoas que ingressaram e que qualificaram as mesmas variáveis no espaço já aromatizado. Os questionários foram sistematizados em uma matriz em Microsoft Excel $2013^{\circledR}$ para, posteriormente, realizar a análise estatística com o IBM SPSS v. 21. Verificou-se a consistência interna com alfa de Cronbach de 0,856 , caracterizou-se a população e estabeleceram-se associações ordinais por meio do coeficiente Gama de Goodman e Kruskal, adicionalmente as possíveis associações foram comprovadas com o teste Qui-quadrado. Um dos resultados mais importantes do estudo demonstrou que o estímulo do sentido do olfato pode ser associado de maneira positiva na percepção das variáveis de atendimento no serviço e na limpeza.

Palavras-chave: marketing; experiencial; sensorial; olfativo; aromas; serviço; persuasão; publicidade; associações.
\end{abstract}




\section{Introducción}

La presencia de los cinco sentidos es de gran importancia en el proceso de producción de significados, los sentidos comúnmente conocidos son el olfato, el gusto, la visión, el oído y el tacto. Otros sentidos son el equilibrio (ubicado en el oído), la temperatura, el dolor y la propiocepción (Derval, 2010), es a través de los sentidos que un cliente puede diferenciar una marca de otra similar (Rupini y Nandagopal, 2015), comunicándose con su entorno, generando percepciones e imágenes mentales acerca de las experiencias que viven con las marcas y el mundo que los rodea, "el marketing da un nuevo giro. El cliente ya no elige un producto o servicio solo por la ecuación costo-beneficio, sino por la vivencia que ofrece antes de la compra y durante su consumo" (Schmitt, 2007, p. 8). Dichas vivencias, en el panorama del marketing y la publicidad, se ven potenciadas con la llegada del marketing sensorial, Krishna (2012) define el marketing sensorial como una estrategia que compromete los sentidos de los consumidores afectando su percepción, juicio y comportamiento. Según Álava, Guerrero y Peña (2009), el ser humano recuerda el $5 \%$ de lo que ve, el $2 \%$ de lo que oye, el $1 \%$ de lo que toca, $15 \%$ de lo que prueba, contra un $35 \%$ de lo que huele, por tal motivo surgen nuevos caminos para comprometer a los públicos dando relevancia a sentidos menos explorados como el olfato que se percibe, entonces, como una estrategia invisible que genera respuestas inconscientes en el comportamiento humano:

Pero el gran crecimiento del marketing olfativo se debe fundamentalmente a su descubrimiento como herramienta de ambientación y comunicación en el punto de venta. El olor es un generador de tráfico, un creador de atmósfera y un símbolo de elevado rendimiento semántico. Fabricantes, distribuidores... diferentes tipos de empresas se asocian en el diseño de estrategias olfativas dirigidas a atraer, sorprender y movilizar al consumidor" (Manzano, Gavilán, Avello, Abril y Serra, 2011, p. 146).

Igualmente, Abril, Gavilán y Serra (2011) plantean que, tanto el sentido olfativo como el auditivo, son involuntarios, lo que quiere decir que siempre están activos, siempre se está oyendo, aunque no se sea consciente de ello; el olfato es una fuerza dinámica e ingobernable que codifica espacios con significado, identifica cuerpos con espacios y puntúa la experiencia temporal del espacio a medida que cambia (Canniford, Riach y Hill 2017).

Cuando las estrategias de marketing se dirigen a los sentidos, este emplea técnicas que influyen en los públicos para lograr aumentar la eficacia de la comunicación y maximizar la experiencia de compra. "El consumidor es cada vez menos leal y tiene más poder, lo que hace que las tiendas se hayan convertido, en parte, en un medio más publicitario de lo que ha sido hasta ahora" (Alfaro, 2012, p. 15).

Por tal motivo es importante plantear algunas preguntas que permitan comprender la importancia del marketing olfativo en la potencialización de experiencias y asociaciones en el ámbito de los servicios, ¿Pueden los aromas, cambiar la percepción del espacio aromatizado? ¿Las variables del servicio pueden verse valoradas de forma diferente al encontrarse expuesto a un aroma? O de igual forma, ¿no existen diferencias significativas en 
la valoración de los espacios y el servicio recibido en un ambiente con un aroma y sin él? Por consiguiente, el objetivo de esta investigación es determinar las asociaciones olfativas en torno a las experiencias del servicio recibido en un fondo de empleados, identificando las diferencias en la percepción del espacio su confortabilidad y limpieza, conociendo la valoración de la atención y el desempeño de los empleados.

\section{Marketing olfativo}

El marketing olfativo utiliza aromas con el fin de evocar emociones e influir en los comportamientos de los compradores y empleados (Ianinni, 2010); Spence, Puccinelli, Grewal y Roggeveen (2014) plantean cómo el olor ambiental puede influir en el comportamiento de los públicos y crear una experiencia más positiva en la interacción marca usuario.

Por medio de los aromas se puede reforzar la notoriedad de la marca y crear una identidad que transmita los valores de la misma, influyendo directamente sobre la percepción de los espacios que se encuentren intervenidos por la estrategia olfativa.

Hoy en día las estrategias de marketing tradicionales van quedando obsoletas dando paso a un mundo de experiencias que conectan las marcas en su interacción con los públicos creando momentos únicos y memorables.

El uso de los cinco sentidos y su importancia para las experiencias de compra y servicio genera la creación de atmósferas únicas y reales con mayor recordación, diferenciación y fidelización, para Rimkute, Moraes y Ferreira (2016) la aplicación de recursos multisensoriales en marketing ha recibido una atención de investigación significativa, ya que las señales sensoriales influyen en los procesos de toma de decisiones de los consumidores al crear asociaciones mentales que fortalecen la recuperación de marcas y productos. Al apelar a los estímulos multisensoriales también brindan múltiples opciones para crear y modificar percepciones como la calidad del producto, ya que un sentido puede generar este tipo de percepciones con mayor fuerza que otros. De esta manera, ha surgido una creciente necesidad por estimular diferentes sentidos, antes no explotados en la comunicación, para lograr acercarse más al público y crear marcas más fuertes en la mente del consumidor, en las que se vea proyectada esa imagen tan anhelada. El sentido del olfato es el sentido que genera mayor nivel de recuerdo y de evocación, esto se debe a que el olor recorre un camino muy corto hasta llegar al denominado sistema límbico, parte baja de nuestro cerebro y es el responsable de las emociones y el recuerdo

Según Palomares (2013), el marketing olfativo se basa en emplear aromas para evocar emociones de los consumidores y así influir en su comportamiento en el punto de venta. El sentido del olfato influye directamente sobre nuestras emociones y recuerdos ya que esta asociado fuertemente con la memoria. Usados de manera efectiva, los aromas pueden producir una respuesta emocional por encima de lo racional, un buen aroma eleva el ánimo y modifica la forma de pensamiento, si se tiene una marca olfativa los clientes la asociaran con la experiencia vivida. 
De todos nuestros sentidos, el más primitivo y más profundamente arraigado es el olfato. Fue con la ayuda de este que nuestros ancestros desarrollaron el gusto por los alimentos, buscaron sus parejas e intuyeron la presencia de los enemigos. Cuando olemos algo, los receptores de la nariz conectan directamente con nuestro sistema límbico, el cual controla las emociones, los recuerdos y la sensación de bienestar. Como consecuencia, la reacción instintiva es instantánea. Así lo explica Pam Scholder Ellen, profesora de marketing de la Universidad Estatal de Georgia: con nuestros demás sentidos, pensamos antes de reaccionar, pero con el olfato, el cerebro responde antes de pensar (Lindstrom, 2009 p. 162).

Ante la importancia que cobra el sentido del olfato en el momento de tomar una decisión de compra, nuevas estrategias de mercado empezaron a tomar fuerza para atraer consumidores en los puntos de venta como factor de éxito en la creación de experiencias y percepción positiva sobre el producto (Labenz, Wiedmann, Bettels $\mathcal{E}$ Haase, 2018) con esta estrategia, es posible crear una marcada diferencia reforzando una imagen positiva en la mente de los públicos, para Hulten (2015), la identidad de marca debe tener coherencia con la materialización de la vivencia de la misma, con la experiencia de marca que se obtiene a través de lo sensorial, así mismo se trata de provocar un estado de relajación y bienestar que facilita la circulación de los públicos por todas las áreas de venta y atención haciéndolos olvidarse de su reloj.

La respuesta inicial y más básica ante un aroma está articulada a las dimensiones de agrado o desagrado, en este punto es vital la intensidad o grado de concentración del aroma y su familiaridad, esto debido a que nuestro cerebro percibe y acepta con mayor facilidad aquellos estímulos con los cuales se siente relacionado. Los efectos de la presentación del producto, fragancias agradables en ambientes apropiados, así como la intención de compra y experiencias placenteras (sensoriales) demuestran que la presentación del producto con la fragancia adecuada genera un efecto más positivo en dichas respuestas (Fiore, Yah y Yoh, 2000); debido a esto es indispensable, al realizar una estrategia olfativa, direccionarla congruentemente con el espacio, producto, marca o perfiles de consumidores.

\section{Aplicaciones del aroma en las estrategias de marketing}

De la misma forma que el logotipo transmite mensajes explícitos e implícitos relacionados con la marca, un odotipo "una forma aromática estable que se inscribe como uno de los elementos distintivos para una marca (Sánchez y Pintado, 2012, p. 291), puede comunicar sensaciones, emociones, valores y recuerdos. Estos aromas denominados odotipos o marcas olfativas, se desarrollan con el objeto de que los públicos los archiven en su memoria olfativa y los asocien al logo o experiencias vividas, el aroma no se debe tomar como un sustituto del logotipo, lo que se busca es una sinergia entre ambos elementos, destinados a reforzar significados o desencadenar ciertas emociones como lo plantea Bonadeo (2005), mientras más canales sensoriales comuniquen la misma idea a la persona que percibe, más fácil le resultará la identificación de esa marca y se fortalecerá su capacidad de memorización. 
Los aromas también pueden estar asociados con la actividad comercial, como por ejemplo el aroma a chicle para las tiendas de niños o limón para artículos de limpieza, olor a crispetas en la sala de cine o todo aquello que pueda incidir de manera estratégica en una decisión de compra o generación de experiencias gratificantes, así mismo pueden reproducir la esencia del producto para incentivar o potenciar su consumo:

En todas estas situaciones, el olor desempeña un papel activo en el proceso de venta. A través del fuerte impacto que tiene en nuestras emociones nos conecta con los recuerdos, activa nuestros deseos, evoca todo tipo de sentimientos de activación o relax, y nos informa o indica adónde dirigir nuestra atención (Manzano et al., 2011, p. 153).

Es el caso de los establecimientos comerciales que llaman la atención de los transeúntes con olor a pan recién horneado, café recién molido o perfumes llamativos en las entradas principales.

La teoría de la congruencia semántica y el marketing sensorial sobre el comportamiento del consumidor sugieren un impacto positivo en el comportamiento de compra, un olor más común, congruente con la categoría, es óptimo, en oposición al aroma congruente y diferenciador del producto (Kivioja, 2017).

En el caso de los servicios su carácter intangible hace que sea difícil para los clientes evaluar una oferta antes de su consumo, al agregar el componente de aroma a sus ofertas de servicio, los proveedores tienen la oportunidad de crear una ventaja competitiva (Goldkuhl, y Styvén, 2007).

\section{Metodología}

La presente investigación tiene un enfoque cuantitativo, de tipo descriptivo de alcance correlacional, cuasiexperimental. Se tomaron de 602 encuestas a personas que ingresaron a las instalaciones del fondo de empleados, se preguntó a 301 personas antes de la intervención (el error relativo en +-5\% para cada toma de muestra) y 301 durante la intervención con aromas, en las cuales se le diligenció un cuestionario estructurado autodiligenciado con escalas de Likert de 1 a 5 que involucraron variables de percepción sobre el espacio y el servicio recibido ( $1=$ Muy en desacuerdo, $2=$ En desacuerdo, $3=$ indiferente, $4=$ De acuerdo, 5= Muy de acuerdo): "Los empleados fueron amables con usted", "Se le atendió de manera oportuna", "Se le ofreció un buen servicio", "Cree que todos nuestros servicios son de calidad", "El ambiente le genera tranquilidad", "Las instalaciones le parecen confortables", "Las instalaciones estaban limpias", "Qué tan rápido se le dio respuesta a su solicitud", "Nuestro desempeño" y "Posibilidad de recomendaciones".

En la primer semana se indagó en la percepción a 301 personas que ingresaron a las instalaciones del fondo de empleados sin intervención de algún esquema de aroma. Para la segunda semana, se hizo una intervención, para ello se utilizó un aspersor de aromas que cubrió toda la sala de atención programado para su funcionamiento dos minutos 
de aspersión y diez minutos de descanso durante las ocho horas de atención al público, utilizando una fragancia desarrollada con aceites esenciales que buscaban llenar el espacio de tranquilidad asociado a un aroma masculino, los componentes de la fragancia desarrollada fueron los siguientes: notas de salida (limón, bergamota), notas medias (nuez moscada, cedro), notas de fondo (sándalo, cedro). Hubo tres notas con cada fragancia, la fragancia debió combinar todas las notas juntas buscando un efecto armonioso para el usuario, la nota de salida, en este caso limón y bergamota, se compuso de la suma de ingredientes que la hizo más ligera, moléculas que se evaporan rápidamente dándole una tonalidad fresca, las notas medias, se componen de moléculas que toman más tiempo para vaporizar, surgen cuando las notas de salida se disipan, la nuez moscada y el cedro calman la ansiedad, la tensión, ayudan a disolver la depresión. La nota de fondo contiene compuestos fijadores, su objetivo principal fue la de mantener el aroma y ayudar a equilibrar los efectos de las otras notas. En este caso el sándalo y el cedro se combinan para tranquilizar. La combinación de la nota de fondo y la nota media decide el tema principal de la fragancia creando una combinación aromática maderosa. De igual forma, durante una semana se recolectaron 301 encuestas a las personas que ingresaron calificando las mismas variables en el espacio ya aromatizado.

Se sistematizaron los cuestionarios en una matriz en Microsoft Excel 2013® para, posteriormente, realizar el análisis estadístico con el IBM SPSS v. 21. Se verificó la consistencia interna con alfa de Cronbach de 0,856 , se caracterizó la población y se establecieron asociaciones ordinales mediante el coeficiente Gamma de Goodman y Kruskal, adicionalmente se comprobaron con la prueba Chi-Cuadrado las posibles asociaciones.

\section{Resultados}

La población se caracterizó así: hombres con un 52,7 \% y mujeres que fueron el 47,3 \% de las personas encuestadas, los rangos de edad se establecieron de la siguiente manera: 18-24 años 1,0 \%, 25 -34 años 9,7 \%, 35-44 años $17,3 \%$, 45 - 54 años 29,7\% y más de 54 años $41,7 \%$. Estado civil: casados $58,0 \%$, solteros $17,3 \%$, unión libre $10,0 \%$, divorciados 7,7 y viudos 7,0 \%. Grado de formación: maestría 30,7 \%, técnicos 20,7 \%, estudios universitarios $20,0 \%$, secundarios $14,0 \%$, primaria $10,0 \%$, doctorado 7,0 \%. Ocupación: jubilado 32,7 $\%$, docente $17,0 \%$, NR, 3,0 \%, otro 2,7\%, personal administrativo $44,7 \%$.

Los asociados del fondo de empleados en su mayoría son casados, con estudios de maestría y hay un alto porcentaje de administrativos y jubilados.

En la figura 1, se aprecian cambios significativos alrededor de la intervención con olor. "Las instalaciones estaban limpias" (con olor), presenta cambios del $35 \%$ en el Muy de acuerdo entre sin olor y con olor. En los otros dos ítems, con una variación de $12 \%$ en la percepción de tranquilidad del ambiente y un 7 \% si les parecía confortable. Aunque no fueron significativos, los incrementos fueron positivos cuando se estuvo expuesto al aroma. 


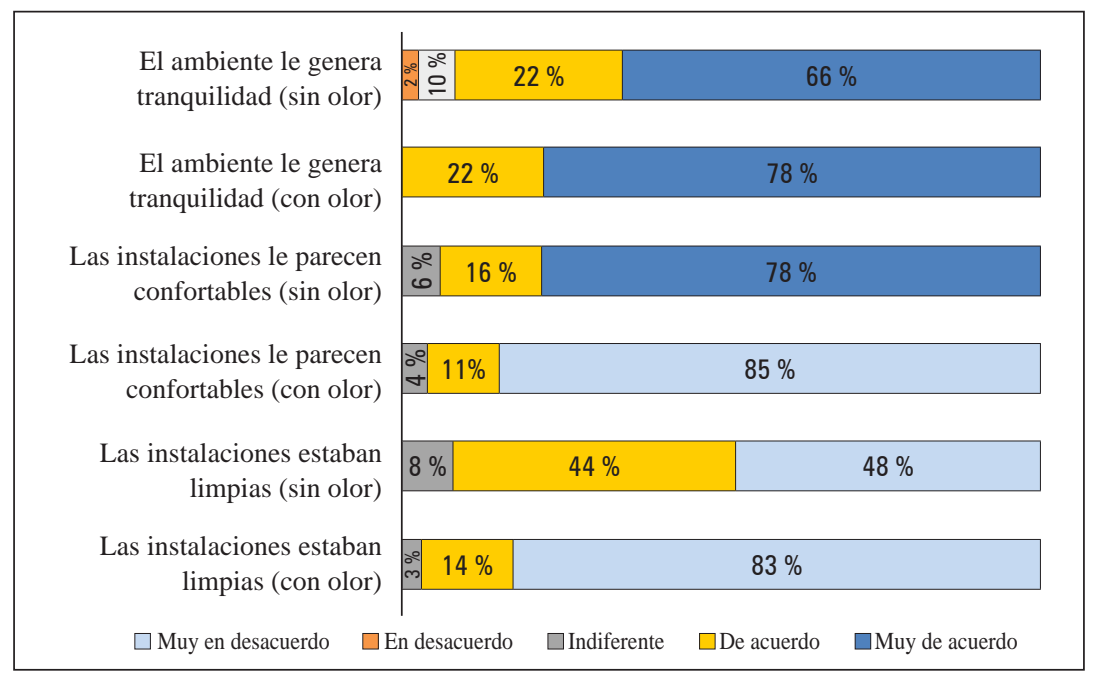

Figura 1. Gráficos de barra apilados comparando la intervención con olor Fuente: elaboración propia

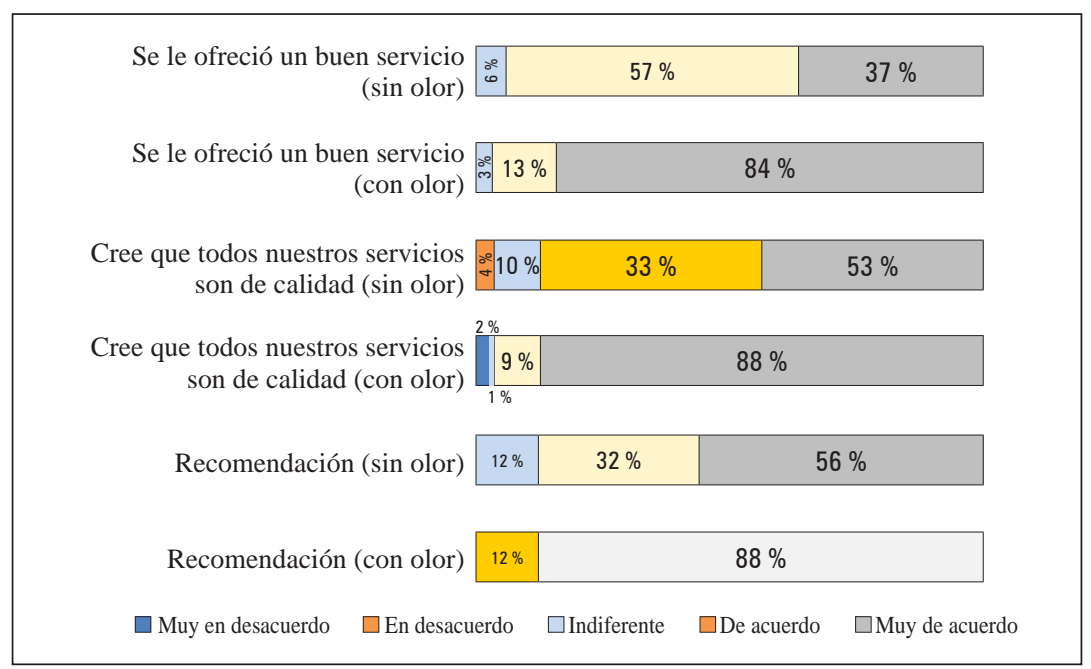

Figura 2. Gráficos de barra apilados comparando la intervención con olor

$$
\text { Fuente: elaboración propia }
$$

En la figura 2 se aprecian variaciones muy significativas alrededor de la intervención con olor. La percepción sobre el buen servicio, pasó de un de 37 \% en el Muy de acuerdo, sin olor, a un 87 \% en muy de acuerdo con olor; para un incremento de un $40 \%$. En cuanto a la calidad del servicio, la variación entre sin olor y olor fue del $35 \%$, seguido de la posibilidad de recomendación con un $32 \%$ de incremento con olor, para unas variaciones de más del $30 \%$ en las tres preguntas. 
En la figura 3, aunque incrementaron, las variaciones no fueron muy significativas alrededor de la intervención con olor. La percepción sobre "Se le atendió de manera oportuna" incrementó en un 11 \% muy de acuerdo con olor, en cuanto a la rapidez de atención incremento en un 13 \% con olor, por último la pregunta del bloque con mayor variación fue sobre la percepción del desempeño, peor, similar o mejor que antes con un $16 \%$ de incremento en muy de acuerdo con olor a mejor que antes.

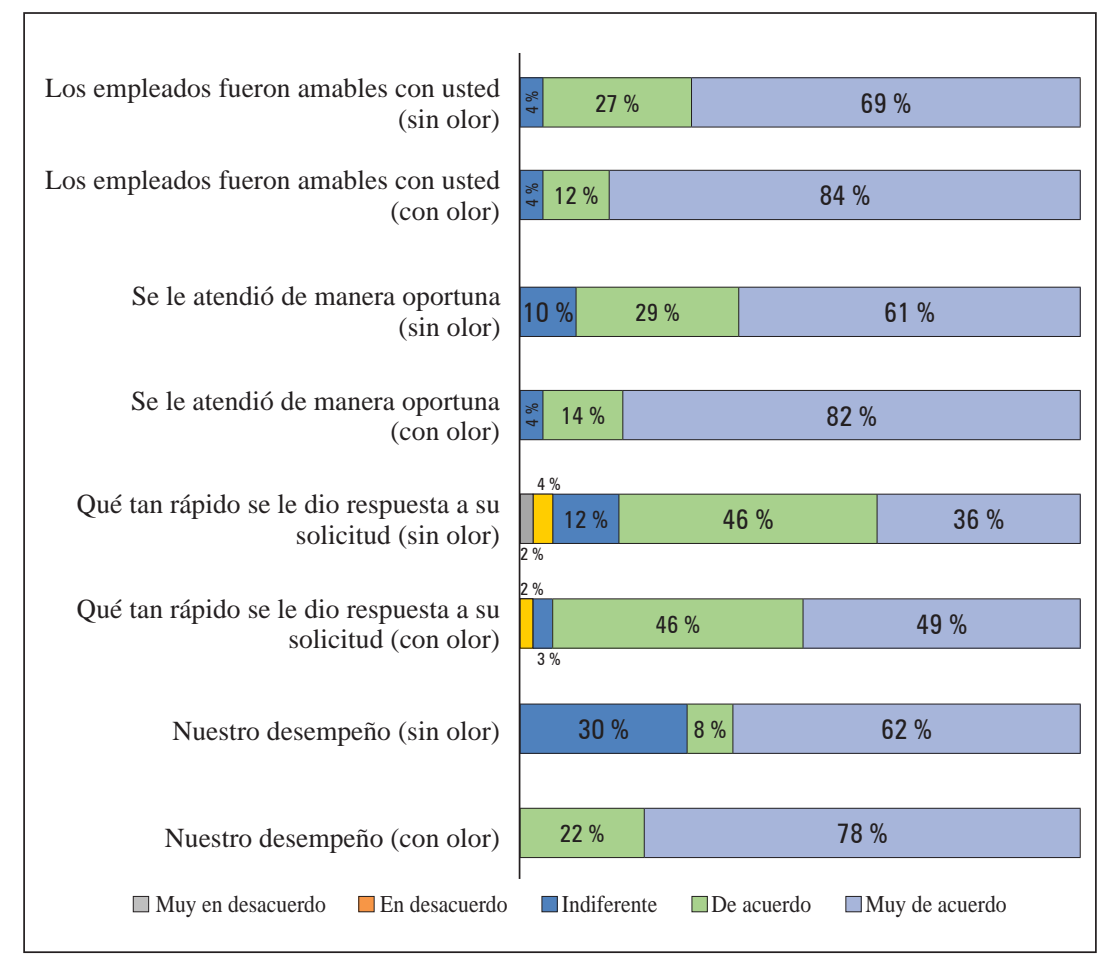

Figura 3. Gráficos de barra apilados comparando la intervención con olor

Fuente: elaboración propia

Se presentaron cambios muy significativos alrededor de la intervención con olor en las variables que involucran la percepción de las instalaciones y su limpieza, en la percepción de tranquilidad del ambiente y confortabilidad existieron variaciones, aunque no fueron significativos los incrementos. Igualmente, la percepción sobre el servicio prestado fue la de mayor variación demostrando que la intervención olfativa es importante si queremos de manera inconsciente incrementar de forma positiva la valoración del servicio.

Aunque no todas las variables se incrementaron en igual proporción, sí existió de manera constante un incremento en la valoración del espacio intervenido por el olor lo que sugiere la importancia de implementar estrategias olfativas como complemento a las estrategias de servicio. Adicionalmente, se comprobó mediante el Gamma de GoodmanKruskal el grado de asociación, para estos niveles de cambio. 
Tabla 1. Asociaciones estadísticas

\begin{tabular}{|c|c|c|c|c|c|c|c|}
\hline & & $\mathrm{Ol}$ & or & & & & \\
\hline Ítems & & Sin olor & Con olor & & Medidas & métricas (Gamm & \\
\hline & & Recuento & Recuento & Valor & $\begin{array}{c}\text { Error típ. } \\
\text { asint. }\end{array}$ & $\mathrm{T}$ aproximada & Sig. Aprox. \\
\hline & 5 & 208 & 252 & 0,367 & 0,084 & 4,106 & 0,000 \\
\hline $\begin{array}{l}\text { Los empleados fueron } \\
\text { amables con usted }\end{array}$ & 4 & 80 & 36 & & & & \\
\hline & 3 & 12 & 12 & & & & \\
\hline & 5 & 183 & 245 & 0,459 & 0,071 & 5,780 & 0,000 \\
\hline $\begin{array}{l}\text { Se le atendió de mane- } \\
\text { ra oportuna }\end{array}$ & 4 & 87 & 43 & & & & \\
\hline & 3 & 30 & 12 & & & & \\
\hline & 5 & 110 & 253 & 0,763 & 0,041 & 13,148 & 0,000 \\
\hline $\begin{array}{l}\text { se le ofrecio un buen } \\
\text { servicio }\end{array}$ & 4 & 172 & 39 & & & & \\
\hline & 3 & 18 & 8 & & & & \\
\hline & 5 & 160 & 263 & 0,682 & 0,054 & 9,790 & 0,000 \\
\hline Cree que todos nues- & 4 & 98 & 27 & & & & \\
\hline tros servicios son de & 3 & 30 & 3 & & & & \\
\hline & 2 & 12 & 0 & & & & \\
\hline & 1 & 0 & 7 & & & & \\
\hline & 5 & 197 & 234 & 0,341 & 0,078 & 4,110 & 0,000 \\
\hline El ambiente le genera & 4 & 67 & 66 & & & & \\
\hline tranquilidad & 3 & 30 & 0 & & & & \\
\hline & 2 & 6 & 0 & & & & \\
\hline
\end{tabular}

Fuente: elaboración propia

Se aprecian las tablas de contingencia (tabla 1) donde se ilustra el grado de asociaciones. Se identificó como el grado de asociación del olor y "Se le ofreció un buen servicio" indicaron un nivel alto (Gamma: 0,763.p<0,000), seguido de "Cree que todos nuestros servicios son de calidad" (Gamma: 0,682.p<0,000) indicando una posible influencia en el servicio y la atención que recibió, aun así las demás asociaciones de la tabla en mención mostraron relaciones moderadas con el olor (las demás Gamma<0,5 pero p<0,000). 
Asociaciones olfativas en torno a la percepción del espacio y el servicio recibido por los asociados a un fondo...

Tabla 2. Asociaciones estadísticas

\begin{tabular}{|c|c|c|c|c|c|c|c|}
\hline \multirow{3}{*}{ Ítems } & & \multicolumn{3}{|c|}{ Olor } & & & \\
\hline & & \multirow{2}{*}{$\begin{array}{l}\text { Sin olor } \\
\text { Recuento }\end{array}$} & \multirow{2}{*}{$\begin{array}{l}\text { Con olor } \\
\text { Recuento }\end{array}$} & \multicolumn{4}{|c|}{ Medidas simétricas (Gamma) } \\
\hline & & & & Valor & $\begin{array}{c}\text { Error típ. } \\
\text { asint. }\end{array}$ & T aproximada & $\begin{array}{l}\text { Sig. } \\
\text { Aprox. }\end{array}$ \\
\hline \multirow{3}{*}{$\begin{array}{l}\text { Las instalaciones le } \\
\text { parecen confortables }\end{array}$} & 5 & 234 & 254 & 0,210 & 0,098 & 2,107 & 0,035 \\
\hline & 4 & 48 & 34 & & & & \\
\hline & 3 & 18 & 12 & & & & \\
\hline \multirow{3}{*}{$\begin{array}{l}\text { Las instalaciones } \\
\text { estaban limpias }\end{array}$} & 5 & 144 & 248 & 0,641 & 0,054 & 9,413 & 0,000 \\
\hline & 4 & 132 & 43 & & & & \\
\hline & 3 & 24 & 9 & & & & \\
\hline \multirow{5}{*}{$\begin{array}{l}\text { Qué tan rápido se le } \\
\text { dio respuesta a su } \\
\text { solicitud }\end{array}$} & 5 & 108 & 148 & 0,314 & 0,066 & 4,568 & 0,000 \\
\hline & 4 & 139 & 137 & & & & \\
\hline & 3 & 36 & 9 & & & & \\
\hline & 2 & 11 & 6 & & & & \\
\hline & 1 & 6 & 0 & & & & \\
\hline \multirow{3}{*}{ Nuestro desempeño } & 5 & 186 & 234 & ,453 &, 066 & 6,101 &, 000 \\
\hline & 4 & 24 & 66 & & & & \\
\hline & 3 & 90 & 0 & & & & \\
\hline \multirow{3}{*}{ Recomendación } & 5 & 168 & 264 & ,713 &, 051 & 9,849 &, 000 \\
\hline & 4 & 96 & 36 & & & & \\
\hline & 3 & 36 & 0 & & & & \\
\hline
\end{tabular}

Fuente: elaboración propia

Similarmente, en la tabla 2 el valor de la "Recomendación" (Gamma=0,713) y "Las instalaciones estaban limpias" generaron una asociación positiva y significativa $(p<0,0000)$ frente a la presencia del olor dentro del estudio. Se observaron asociaciones muy débiles frente "Las instalaciones le parecen confortables" (Gamma=0,210); no obstante, las demás relaciones fueron moderadas frente a "Qué tan rápido se le dio respuesta a su solicitud".

En conclusión, frente a las asociaciones y cambios porcentuales, existe una posible influencia o comprensión del olor desde la percepción del servicio y la limpieza, mas no desde lo confortable y lo amable en el trato. 


\section{Discusión}

Los sentidos se han convertido en un factor determinante en el momento de elegir una marca y transmitir una imagen positiva. Cada vez la publicidad se enfoca más en estimular al público objetivo por medio de olores, sabores, colores, formas, sonidos, texturas, etc., Krishna, Cian y Aydinoğlu, (2017) señalan que los aspectos sensoriales del diseño de empaques afectan las etapas clave de la experiencia del cliente y procuran generar experiencias positivas y memorables en los públicos los cuales buscan atributos diferenciales e innovadores en los productos y servicios que apropian a diario. Piqueras y Spence (2012) sugieren que los envases pueden atraer a los clientes con sus componentes hápticos, en una investigación realizada los participantes degustaron galletas en envases, con variadas texturas superficiales (rugosas/granulares vs. lisas), los resultados mostraron que los participantes calificaron las muestras de alimentos del empaque rugoso significativamente más crujiente y duro que aquellos del recipiente liso. Así, Alcántara, Artacho, Zamora y Martínez (2014) aplicaron un estudio semántico diferencial para investigar la influencia de los canales sensoriales en la estructura semántica de la percepción. El estudio constó de cuatro experimentos en los que los voluntarios informaban su percepción de una muestra de estímulos visuales, acústicos, olfativos y táctiles; se obtuvo el espacio semántico para cada sentido analizando las diferencias de estructura y significado. Los resultados evidenciaron que los conceptos que utilizan las personas para expresar la percepción dependen del sentido utilizado, otros autores mencionan la implementación de una tira perfumada en anuncios de perfumería para apelar al sentido olfativo o el uso de elementos hápticos para proporcionar una experiencia específica del tacto.

Haase, Wiedmann y Bettels (2018) realizaron una investigación para validar cómo cambiaría la percepción de un anuncio al usar imágenes sensoriales, los hallazgos sugieren que la modificación de un medio de publicidad puramente visual en forma de elementos complementados que mejoren las imágenes sensoriales es un enfoque valioso para excitar otros sentidos que afecta positivamente a la percepción del consumidor en términos de diseño del producto percibido y actitud hacia la compra.

Por otra parte, Laninni (2010) nos relata las estrategias que tomó el centro comercial Miramar de Fuengirola (Málaga): implementó distintos aromas florales que transmitían al cliente una sensación de bienestar y tranquilidad, como los de rosas, jazmín, gardenia, dama de noche, azahar, arboleda y césped. Resultados similares obtuvieron Lehrner, Marwinski, Lehr, Johren y Deecke (2005) al aromatizar la sala de espera de un consultorio odontológico con un aroma cítrico disminuyendo así los niveles de ansiedad, incrementando el nivel de calma y mejorando el estado del ánimo y relajación de los pacientes. Igualmente se evidencian otros resultados positivos en la implementación de estrategias olfativas, por ejemplo, Schifferstein y Blok (2002) demostraron que, aunque el aroma a limón en una pizzería no generó efectos particulares sobre las personas, el de lavanda sí tuvo un efecto en el gasto de dinero y en el tiempo de permanencia a pesar de no estar relacionado con el espacio o el producto. 
Hirsch (1995) realizó una aromatización en dos secciones de un casino incrementando en un 45 \% las apuestas en las máquinas de las zonas aromatizadas y Liljenquist, Zhong y Galinsky (2010), encontraron una variación en la intención de consumo de un producto o gasto de dinero al implementar un aroma en un espacio comercial. Así mismo, otro hallazgo de la investigación fue en torno a las asociaciones entre el aroma y la calidad del servicio recibido tal y como lo plantean Chebat y Michon (2003) indagando a 145 personas que se encontraban dentro de un centro comercial y encontraron una percepción favorable sobre el entorno y sobre la calidad de los productos.

\section{Conclusiones}

Los resultados del estudio demuestran que la estimulación del sentido del olfato tiene una gran influencia en la percepción de la calidad del servicio y aseo de las instalaciones.

La fragancia aromática maderosa, hace sentir a las personas en un estado de tranqui lidad y disminuye la agresividad en los entornos comerciales.

La utilización de los aromas en los puntos de contacto con los públicos, fortalece la imagen de los productos ante los ojos de los mismos, de igual manera la prestación de los servicios es mejor valorada, debido al poder de los aromas en la transformación de un espacio ordinario en una atmósfera llena de tranquilidad y placer.

Es importante realizar las estrategias olfativas de una forma coherente y articulada con la estrategia de la marca para que estas sean eficaces y no causen una experiencia negativa en los públicos.

Realizar estrategias olfativas siempre será una buena alternativa para incrementar la percepción positiva en cada una de las etapas del ciclo del servicio en las cuales se tiene contacto con los públicos.

Al realizar estrategias olfativas buscando incidir directamente sobre la venta del producto, es recomendable potencializar los aromas asociados con la categoría. Así mismo, el desarrollo de marcas olfativas busca impulsar a largo plazo la recordación de la marca o las experiencias vividas en torno al proceso de compra o consumo.

Los aromas cítricos, como el limón, generan percepciones positivas sobre la limpieza del espacio intervenido, de igual forma el olor a nuez moscada y cedro disminuye la ansiedad aumentando el estado de ánimo de las personas.

Al igual que investigaciones anteriores, la aromatización de los espacios comerciales o de contacto con los públicos, incrementa la percepción de calidad de los productos o servicios.

Al intervenir el espacio con un aroma, se incrementan de manera positiva las variables asociadas con los atributos de los productos o servicios ofertados. 


\section{Referencias}

Álava, L., Guerrero, M. y Peña, B. (2009). Aplicación del marketing olfativo en las cadenas de supermercados de Guayaquil (Tesis de pregrado). Escuela Superior Politécnica del Litoral, Guayaquil, Ecuador.

Alcántara, E., Artacho, M., Zamora, T. y Martínez, N. (2014). Exploratory Study of the Influence of the Sensory Channel in Perception of Environments. Journal of Sensory Studies, 29(4), 258-271. doi:10.1111/joss. 12099

Alfaro, E. (2012). El ABC del shopping experience. Cómo generar experiencias para vender más. Madrid: Wolters Kluwer.

Bouzas, D. G., Barrie, C. A., E Serra, T. (2011). Marketing olfatorio: el olor de los deseos. Harvard Deusto Marketing y Ventas, (103), 34-39.

Bonadeo, M. (2005). Odotipo: historia natural del olfato y su función en la identidad de marca (Tesis doctoral). Universidad Austral, Buenos Aires, Argentina.

Canniford, R., Riach, K. y Hill, T. (2017). Nosenography: How smell constitutes meaning, identity and temporal experience in spatial assemblages. Sage Journals, 18(2), 234-248. doi: $10.1177 / 1470593117732462$

Chebat, C. y Michon, R. (2003). Impact of ambient odors on mall shoppers' emotions, cognition, and spending: A test of competitive causal theories. Journal of Business Research, 56(7), 529-539. doi:10.1016/S01482963(01)00247-8

Derval, D. (2010). The Right Sensory Mix. Berlín: Springer-Verlag.

Fiore, A., Yah, X. y Yoh, E. (2000). Effects of a product display and environmental fragrancing on approach responses and pleasurable experiences. Psychology y Marketing, 17(1), 27-54. doi.org/10.1002/ (SICI) 1520-6793(200001) 17:1 <27::AID-MAR3 > 3.0.CO;2-C

Goldkuhl, L. y Styvén, M. (2007). Sensing the scent of service success. European Journal of Marketing, 41(11-12), 1297-1305. doi:10.1108/03090560710821189

Haase, J., Wiedmann, K. y Bettels, J. (2018). Sensory imagery in advertising: How the senses affect perceived product design and consumer attitude. Journal of Marketing Communications, 15(1), 2-3. doi: $10.1080 / 13527266.2018 .1518257$

Hirsch, A. (1995). Effects of ambient odors on slot-machine usage in a Las Vegas Casino. Psychology and Marketing, 12, 585-594. doi: 10.1002/ mar.4220120703

Hulten, B. (2015). Marketing sensorial: el concepto de experiencia de marca multisensorial. European Business Review, 23(3), 256-273. doi.org/10.1108/09555341111130245

Ianinni, M. (2010). Marketing Olfativo un valor diferencial. MK: Marketing + ventas, 253(2010), 58-64.

Kivioja, K. (2017). Impact of point-of-purchase olfactory cues on purchase behavior. Journal of Consumer Marketing, 34(2), 119-131. doi:10.1108//CM-08-2015-1506

Krishna, A. (2012). An integrative review of sensory marketing: Engaging the senses to affect perception, judgment and behavior. Journal of Consumer Psychology, 22, 33-351. doi: 10.1016/j.jcps. 2011.08 .003

Krishna A., Cian, L. y Aydınoğlu, N. (2017). Sensory Aspects of Package Design. Journal of Retailing, 93(1), 43-54. doi.org/10.1016/j.jretai.2016.12.002 
Labenz, F., Wiedmann, K. P., Bettels, J., \& Haase, J. (2018). Sensory Stimuli in Print Advertisement â€"Analyzing the Effects on Selected Performance Indicators. Journal of International Business Research and Marketing, 3(2), 7-15.

Lehrner, J., Marwinski, G., Lehr, S., Johren, P. y Deecke, L. (2005). Ambient odors of orange and lavender reduce anxiety and improve mood in a dental office. Physiology and Behavior, 86(1-2), 92-95. doi: 10.1016/j.physbeh.2005.06.031

Lindstrom, M. (2009). Compradicción. Verdades y mentiras de por qué las personas compran. Bogotá: Norma, 248p.

Liljenquist, K., Zhong, C. y Galinsky, A. (2010). The smell of virtue. Clean scents promote reciprocity and charity. Psychological Science, 21, 381-383. doi:10.1177/0956797610361426

Manzano, R., Gavilán, D. Avello, M., Abril, C. y Serra, T. (2011). Marketing sensorial: comunicar con los sentidos en el punto de venta. Madrid: Prentice Hall.

Palomares, R. (2013). Marketing en el punto de venta: comunicación y promoción. Madrid: ESIC.

Piqueras-Fiszman, B. y Spence C. (2012). The Influence of the Feel of Product Packaging on the Perception of the Oral-somatosensory Texture of Food. Journal of Food Quality and Preference, 26(1), 67-73. doi:10.1016/j.foodqual.2012.04.002

Rimkute, J., Moraes, C. y Ferreira, C. (2016). The effects of scent on consumer behaviour. International Journal of Consumer Studies, 40(1), 24-34. doi:10.1111/ijcs.12206

Rupini, R.V. y Nandagopal, R. (2015). A Study on the Influence of Senses and the Effectiveness of Sensory Branding. Journal of Psychiatry, 18(2) 236. doi:10.4172/Psychiatry. 1000236

Sánchez, J. y Pintado, T. (2012). Nuevas tendencias en comunicación. Madrid: ESIC.

Schifferstein, H. y Blok, S. (2002). The signal function of thematically (in) congruent ambient scents in a retail environment. Chemical Senses, 27(6), 539-549. Doi:10.1093/chemse/27.6.539

Schmitt, B. (2007) Experiential Marketing. Barcelona: Deusto.

Spence, C., Puccinelli N., Grewal D. y Roggeveen, A. (2014). Store Atmospherics: A Multisensory Perspective. Journal of Psychology and Marketing, 31(7), 472-488. doi: 10.1002/mar.20709 